\title{
Developmental Changes in the Visual Acuity of Red Sea Bream Pagrus major
}

\author{
Yasushi Shiobara, Seiji Akiyama, and Takafumi Arimoto \\ Department of Marine Science and Technology, Tokyo University of Fisheries, \\ Konan, Minato, Tokyo 108-8477, Japan
}

(Received May 18, 1998)

\begin{abstract}
Developmental changes in the visual acuity of red sea bream were investigated by histological examination of the retina for 61 individuals from juvenile to adult stage (BL 23-609 mm). The highest cone density could be located in the dorso-temporal area of the retina. The visual acuity depends both on the eye lens diameter and the cone density on the retina. The previous study reported that the minimum separable angle is obviously greater than the resolving power of the lens. The lens diameter increased from $1.3 \mathrm{~mm}$ to $11.8 \mathrm{~mm}$ proportionally with the growth of body length. The cone density was found to fit in an exponentially declining curve, where it steeply decreases in the range of 23-100 mm $B L$ and becomes a gentle slope to be around $200-400$ cells $/ 0.01 \mathrm{~mm}^{2}$ for samples over $100 \mathrm{~mm} B L$. According to the results, the visual acuity was increased with growth; from 0.05 for $23 \mathrm{~mm} B L$ to 0.28 for $504 \mathrm{~mm} B L$ specimen.

The improvement of acuity can be attributed increase of the lens focal length rather than of the cone density. The visual acuity (V.A.) was shown to fit to the allometric function of the body length $(B L)$ as follows,

$$
V . A .=0.00711 B L^{0.588}, \quad\left(r^{2}=0.964\right) .
$$

Key words: red sea bream, visual acuity, visual axis, growth, development, retinal morphology, cone cell
\end{abstract}

The gear recognition of the target species can be an important factor for understanding the capture process of the fishing gear. In order to design the selective fishing gear and methods, the size differences in visual acuity of target and un-target species is one of the keys to explain how the fish react the gear. The visual acuity of an animal is a measure of its capacity to resolve fine details in the field of view, ${ }^{1)}$ and it can be expressed as the minimum separable angle in discriminating nearest two visual targets. Previous research works demonstrated the development of higher visual acuity with growth for herring Clupea harengus ${ }^{2)}$ and walleye pollock Theragra chalcogramma $a^{3}$ through histological examinations, while for plaice Pleuronectes platessa, turbot Scophthalmus maximus, ${ }^{4}$ and rainbow trout Salmo gairdneri ${ }^{5)}$ through the both methods of histological and behavioral examinations. The minimum visible angle can be also measured as the reactive distance to visual target by behavioral method. The minimum visible angle as the visual acuity was reported to increase with growth for striped beakperch Oplegnathus fasciatus ${ }^{6}$ ) and bluegill sunfish Lepomis macrochirus. ${ }^{7}$

In this paper, the visual acuity of red sea bream $P$. major was examined with special reference in the developmental change with growth as the function of fish size ranged from $23 \mathrm{~mm}$ to $609 \mathrm{~mm}$ body length by histological observation of the retina.

\section{Materials and Methods}

Sixty-one individuals in total were obtained from the different locations of Japan, of which 20 specimens of 23$117 \mathrm{~mm}$ body length $(B L)$ from the Kanagawa Mariculture
Center, and 34 specimens of $119-358 \mathrm{~mm} \mathrm{BL}$ form a local fish farmer in Ishikawa prefecture. The other four specimens of 322-609 mm were collected from the catch of set net in Ibaraki prefecture, and another 3 specimens of 245$270 \mathrm{~mm}$ at local fishmongers in Tokyo. The lens diameters were measured to the nearest $0.1 \mathrm{~mm}$, then the retina samples were prepared for the microscopic observation by the microtome tangential dissection in $4 \mu \mathrm{m}$ thickness. In order to locate the region of the highest cone density, the retina of two specimens ( $B L 245 \mathrm{~mm}$ and $B L 609 \mathrm{~mm}$ ) were divided into 21 parts for tangential sections. Two types of cone were observed; the twin and the single cone. In this case, the both types of cone were counted together for the calculation of the cone density. According to the distribution pattern of cone cells for two specimens, the tangential sections for the other specimens were made only for the dorso-temporal region as the highest density.

The minimum separable angle was determined from the highest density of cones per $0.01 \mathrm{~mm}^{2}$ area in each retinal region using the equation given by Tamura ${ }^{8)}$ as follows;

$$
\alpha_{\text {rad }}=\frac{1}{F} \times\left[\frac{2 \times 0.1 \times(1+0.25)}{\sqrt{n}}\right],
$$

where $\alpha_{\text {rad }}$ is the minimum separable angle in radians, $F$ is the focal length of the lens which can be calculated from Matthiesson's ratio (2.55 times of the lens radius) ${ }^{8)}$ and the constant value of 0.25 is the degree of shrinkage through the histological procedure, and $n$ is the number of cones per $0.01 \mathrm{~mm}^{2}$ identified through the microscopic observation. Hence, the visual acuity $(V . A$.$) in degree is the$ inverse of the minimum separable angle $\left(\alpha_{r a d}\right)$, as follows; 


$$
\text { V.A. }=\left(\alpha_{\text {rad }} \times \frac{180}{\pi} \times 60\right)^{-1}
$$

\section{Results}

Figure 1 shows the contour map of the number of cones per $0.01 \mathrm{~mm}^{2}$ area for specimens of $245 \mathrm{~mm}$ and $609 \mathrm{~mm}$ $B L$. The maximum cone densities of 292 cells $/ 0.01 \mathrm{~mm}^{2}$ for the smaller fish and 198 cells $/ 0.01 \mathrm{~mm}^{2}$ for the larger fish were determined in the dorso-temporal area of the retina, which indicates the visual axis is the lower-fore direction, as suggested both by Tamura ${ }^{8)}$ and Kawamura et $a{ }^{9}{ }^{9)}$ for the fish.

The changes in the lens diameter and the number of cones are shown in Fig. 2A and Fig. 2B respectively. The relationship between the lens diameter and the body length can be expressed by the allometric regression in Fig. 2A, which power equation can be useful to explain the partial body growth against the whole body growth.$^{10}$ The lens diameter increases in proportion with growth, while the cone density shows a decreasing tendency. The lens diameter $(D)$ according to the body length $(B L)$ was revealed from an allometric regression analysis, as being expressed by the following equation:

$$
D=0.138 B L^{0.717}, \quad\left(r^{2}=0.977\right) .
$$

With the same procedure of an allometric function analysis, Zaunreiter et al. ${ }^{11)}$ reported the relationship between the lens growth and the body length of cyprinid fishes.

The cone density when being plotted against the body length in Fig. 2B was found to fit in an exponentially declining curve, where it steeply decreases in the range of 23-100 $\mathrm{mm} B L$ and becomes a gentle slope to be around
200-400 cells $/ 0.01 \mathrm{~mm}^{2}$ for samples over $100 \mathrm{~mm} B L$. The visual acuity or minimum separable angle for visual target can be defined as the function of the resolving powers of the retina and lens.

In the present study, even with the decrease in the cone density in Fig. 2B, the growth effect of the lens dominates higher visual acuity with body length increase as shown in Fig. 2C. A regression analysis suggests an allometric relationship of the visual acuity $(V . A$.$) against the body$ length $(B L)$ with the high correlation coefficient as expressed by the following equation:

$$
\text { V.A. }=0.00711 B L^{0.588}, \quad\left(r^{2}=0.964\right) .
$$

\section{Discussion}

In the present study, we suggest the equation (2) for determining the visual acuity of $P$. major from the body length, for the range from $23 \mathrm{~mm}$ to $609 \mathrm{~mm}$. The visual acuity for the minimum specimen of $23 \mathrm{~mm} B L$ in this report was 0.045 . Concerning the visual acuity for the larval stage of Pagrus species, Pankhurst ${ }^{12)}$ reported the results of minimum separable angle for Pagrus auratus as $2^{\circ} 10 \mathrm{~min}$. for $3.2 \mathrm{~mm}$ standard length, and $52 \mathrm{~min}$. for $5.8 \mathrm{~mm} \mathrm{SL}$, determined morphologically from the retinal cone density, which were equivalent to 0.0077 and 0.0192 as the visual acuity, respectively. The equation (2) for the visual acuity of red sea bream $P$. major gives the very close value as 0.0200 for $5.8 \mathrm{~mm} B L$, while rather the overestimation as 0.0141 for $3.2 \mathrm{~mm} B L$. The discussion on the appropriateness of the allometric growth model for the visual acuity can not be completed because no larval stage samples nor larger samples over $609 \mathrm{~mm} B L$ for $P$. major were available in this report. For the further discussion,

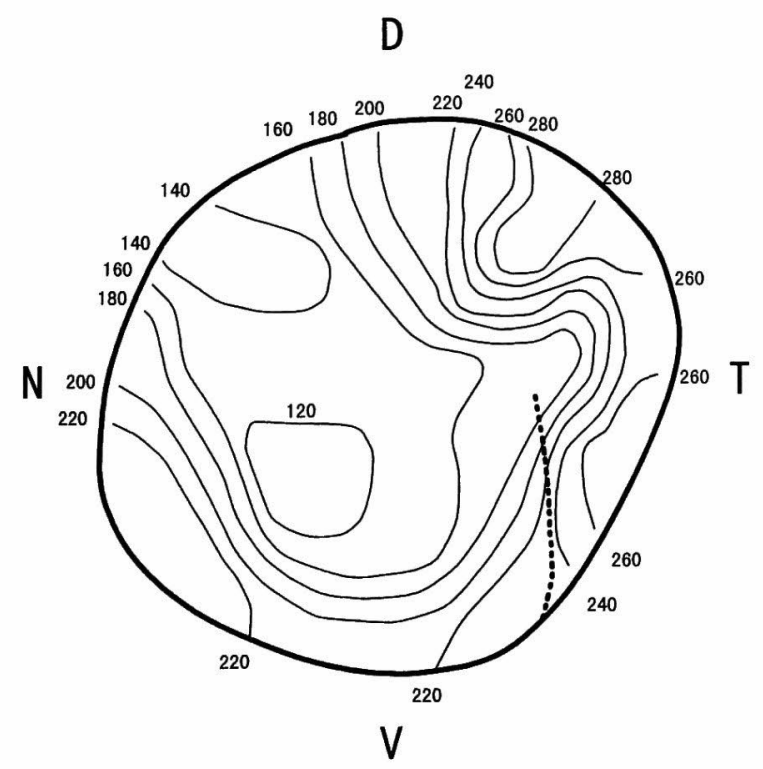

(B.L.245mm)

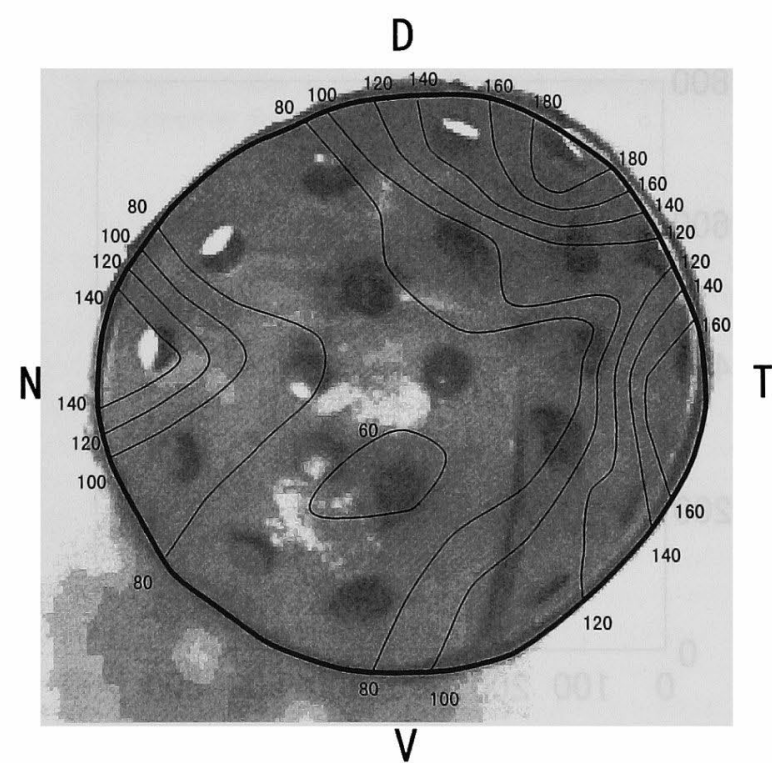

(B.L.609mm)

Fig. 1. Contour map of cone density in red sea bream for two specimens (body length $245 \mathrm{~mm}$ and $609 \mathrm{~mm}$ ).

The number of cones in $0.01 \mathrm{~mm}^{2}$ was counted in the tangential se4ctions and its distribution pattern was shown by iso-density curves. The broken line on the left figure ( $B L 245 \mathrm{~mm}$ ) indicates the optic cleft. The right figure ( $B L 609 \mathrm{~mm})$ shows the overlapped image with the photograph of the whole sampled retina which indicates the optic cleft and cut holes clearly. D, dorsal; T, temporal; V, ventral; N, nasal. 
the wider range of samples for $P$. major will be required. However, with higher correlation coefficient between the body length and the visual acuity, the equation (2), can be available for the most of the commercial size in this species. According to the equation (2), the visual acuity of $200 \mathrm{~mm} B L$ specimen was 0.160 , which is nearly equal to the result obtained by Tamura as to be 0.156 for the same body length. ${ }^{8)}$

The results on the higher visual acuity with growth were also reported for herring ${ }^{2)}$ and perch ${ }^{13)}$ which was attributed to the increase of focal length due to the lens diameter growth. These comparisons of the developmental changes of visual acuity with growth among species can be related

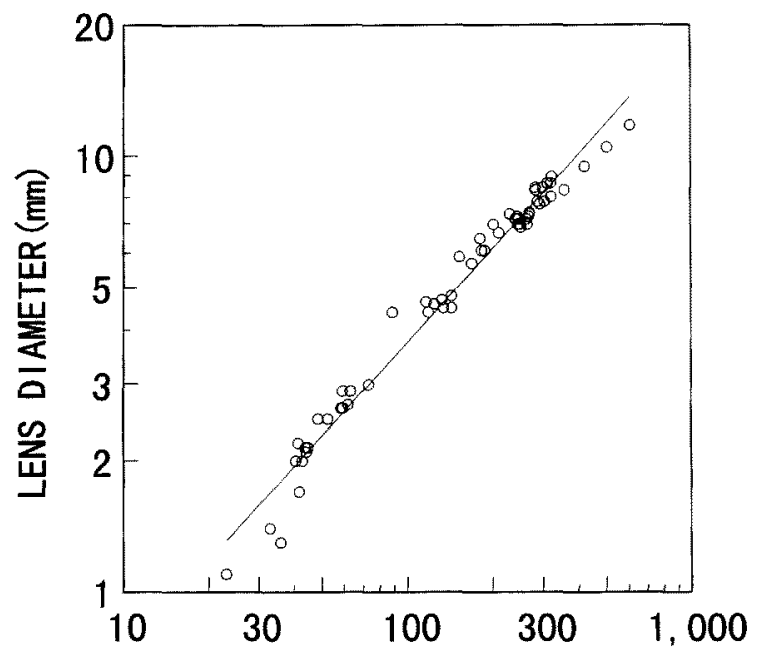

BODY LENGTH (mm)

A

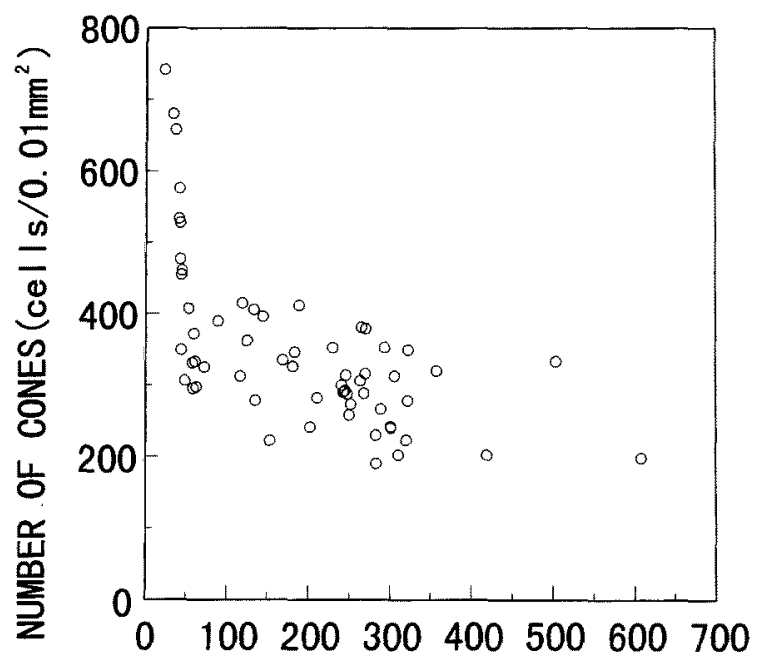

BODY LENGTH $(\mathrm{mm})$

B to the ecological adaptation such as the specific characteristics of feeding behavior. Kawamura et al. ${ }^{9)}$ reported the developmental change of visual cell morphology of larvae and juveniles for red sea bream in relation to the adaptive changes with the shift from pelagic to benthic habitats. The size range of specimens in this report is slightly larger, so that the developmental change of visual acuity with growth can be related to the searching and locating ability on the food organisms in the benthic habitats. The highest visual acuity observed in the present study was 0.284 for $504 \mathrm{~mm} B L$ specimen, which can be equivalent to the minimum separable angle as $3.52 \mathrm{~min}$. It should be noted that this value is higher than 27 species of coastal fishes examined by Tamura, ${ }^{8}$ while it is lower than the large pelagic species by Tamura and Wisby, ${ }^{14)}$ as well as tunas and marlins which were examined by Kawamura et al ${ }^{15)}$

The retinal morphology such as the cone density and its spacial arrangement can determine the ultimate limit to visual resolution. ${ }^{16)}$ The equation (2) given by Tamura ${ }^{8)}$ defines the morphological acuity according to the angle subtended at the nodal point of the eye by two adjacent visual cone cells. Determination of the visual acuity through the behavioral examination, however, resulted in lower values than morphologically determined acuity, even for the same sizes of same species. ${ }^{17}$ ) There are some discussions for explaining this discrepancy between the two estimated acuities. One possible interpretation is that the morphological acuity by the cone density does not take into account the convergence of neural output from the cones to the ganglion cells as the higher ordered image processing. ${ }^{18)}$

For discussing the visual recognition of the gear in the capture process, the developmental change of visual acuity with growth can suggest the possible differences of reacting distance against the gear. The differences in visual acuity between the minimum value of 0.045 in $23 \mathrm{~mm} \mathrm{BL}$ and

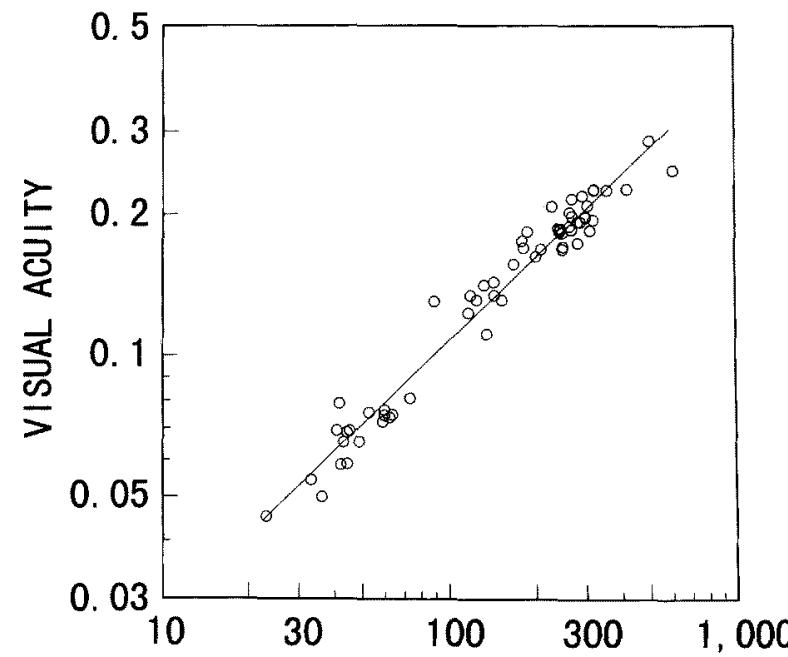

BODY LENGTH $(\mathrm{mm})$

C

Fig. 2. Relationship between optical parameters and body length in Pagrus major.

A: lens diameter, B: cone density, C: visual acuity. Increase tendency of the lens diameter dominates the higher visual acuity with the body length, even with the decrease tendency of the cone density. 
the maximum of 0.284 in $504 \mathrm{~mm} B L$ individuals imply the 6.3 times differences in visual perception for recognizing the fine details of the gear. If the larger fish have the higher ability to detect the approaching gear such as trawl nets from the further distance, they can be allowed to have a longer time for avoiding gear. This sort of information on the scale effect of visual perception, together with the swimming ability, can offer the new approach for the size selectivity in the trawl net. The visual perception of the gear can be influenced by the optical characteristics of the overall gear construction as well as the materials of gear components such as the floats, ropes, twine and netting against the background of water in the different ambient light intensity, transparency, depth and time of day. ${ }^{19)}$ The results by the histological examination in this paper can give the maximum ability for the gear recognition in the ideal light conditions. Further studies will be required through the behavioral observations to measure the visual acuity how the fish detect the fine visual target.

Acknowledgments We are indebted to HOKUMO Co., Ltd. for providing the opportunities to collect the large size samples during the set- net operation in Ibaraki Prefecture, as well as the wide size range from the farming site in Ishikawa Prefecture.

\section{References}

1) W. R. A. Muntz: Comparative aspects in behavioral studies of vertebrate vision, in "Comparative physiology" (ed. by H. Davson \& L. T. Graham Jr.), Academic Press, New York, 1974, pp. 255-261.

2) J. H. S. Blaxter and M. P. Jones: The development of the retina and retinomotor response in the Herring. J. Mar. biol. Ass. U.K., 47, 677-697 (1967).

3) X. M. Zhang and T. Arimoto: Visual physiology of Walleye pollock in relation to capture by trawl nets. ICES mar. Sci. Symp., 196, 113-116 (1993)

4) D. A. Neave: The development of visual acuity in larval Plaice and Turbot. J. Exp. Mar. Biol. Ecol., 78, 167-175 (1984).

5) H. Rahmann, G. Jeserich, and I. Zeutzius: Ontogeny of visual acuity of Rainbow trout under normal conditions and light deprivation. Behaviour, LXVIII, 315-322 (1978).

6) T. Miyazaki: The change of visual acuity of larvae and juveniles of striped beakperch in accordance with their growth. Nippon Suisan Gakkaishi, 59, 437-444 (1993) (in Japanese)

7) J. E. Breck and M. J. Gitter: Effect of fish size on the reactive distance of Bluegill sunfish. Can. J. Fish. Aquat. Sci., 40, 163-167 (1983).

8) T. Tamura: A study of visual perception in fish, especially on resolving power and accommodation. Nippon Suisan Gakkaishi, 22, 536557 (1957).

9) G. Kawamura, R. Tsuda, H. Kumai, and S. Ohashi: The visual cell morphology of Pagrus major and its adaptive change with shift from pelagic to benthic habitats. Nippon Suisan Gakkaishi, 50, 1975-1980 (1984).

10) R. E. Strauss and L. A. Fuiman: Quantitative comparison of body form and allometry in larval and adult Pacific sculpins. Canad. $J$. Zool., 63, 1582-1589 (1985)

11) M. Zaunreiter, H. Junger, and K. Kotrschal: Retinal structure; physiology and pharmacology: Retinal morphology of cyprinid fishes: A quantitative histological study of ontogenetic and interspecific variation Vision Res., 31, 383-394(1991).

12) P. M. Pankhurst: Age-related changes in the visual acuity of larvae of New Zealand snapper, Pagrus auratus. J, mar, biol. Ass. U.K., 74, 337-349 (1994)

13) S. A. Guma'a: Retinal development and retinomotor response in perch, J. Fish Biol., 20, 611-618 (1982).

14) T. Tamura and W. J. Wisby: The visual sense of pelagic fishes especially the visual axis and accommodation Bull. Mar. Sci. Gulf Caribbean, 13, 433-448 (1963)

15) G. Kawamura, W. Nishimura, S. Ueda, and T. Nishi: Vision in Tunas and Marlins. Mem. Kagoshima Univ. Res. Center S. Pac., 1, 3-47(1981).

16) R. H. Douglas and C. W. Hawryshyn: Behavioral studies of fish vision, in "The visual system of fish" (ed. by R. H. Douglas \& M. B. A. Djamgoz), Chapman and Hall, London, 1990, pp. 373-407.

17) P. M. Pankhurst, N. W. Pankhurst, and J. C. Montgomery: Comparison of behavioral and morphological measures of visual acuity during ontogeny in a teleost fish. Brain Behav. Evol., 42, 178-188 (1993).

18) H. 1. Browman, W. C. Gordon, B. I. Evans, and W. J. O'Brien: Correlation between histological and behavioral measures of visual acuity in a zooplanktivorous fish, the White crappi. Brain Behav. Evol., 35, 85-97 (1990).

19) C. W. Glass, C. S. Wardle, S. J. Gosden, and D. N. Racey: Studies on the use of visual stimuli to control fish escape from codends. I. Laboratory studies on the effect of a black tunnel on mesh penetration. Fisheries Res., 23, 157-164 (1995). 